\title{
Gregorio de Tours y la hagiografía del siglo vl. Aproximaciones histórico-teológicas al libro Sobre las virtudes de san Martín"
}

\author{
Gregory of Tours and Hagiography in the Sixth Century. \\ Historical and Theological Approaches to the Book \\ About the Virtues of Saint Martin
}

\author{
Marcelo AGUIRRE DURÁN \\ Universidad de los Andes, Santiago de Chile \\ https://orcid.org/0000-0002-6282-5847 \\ marceaguirred@gmail.com
}

Resumen: El obispo Gregorio de Tours († 594) redactó ocho libros hagiográficos, cuatro de los cuales fueron dedicados a los milagros realizados por san Martín -De uirtutibus sancti Martini episcopi-. Esta recopilación de favores, poco difundida en el mundo hispanohablante, representa un esfuerzo pedagógico-moral y permite comprender el proyecto pastoral de este escritor galorromano del siglo VI. El presente trabajo intenta mostrar cómo la labor hagiográfica de este letrado obispo posibilita el análisis de las particularidades históricas de una sociedad eminentemente religiosa, en la cual la transmisión de los ideales de una disciplina de vida evangélica y la propagación de la devoción a san Martín resultan centrales.

Palabras clave: Gregorio de Tours; Martín de Tours; hagiografía; milagros.

\begin{abstract}
Keywords: Gregory of Tours; Martin of Tours; hagio-
graphy; miracles.

Abstract: Bishop Gregory of Tours († 594) wrote e no uirtutibus sancti Martini episcopi-. This collection of favours, little known in the Spanish-speaking world, represents a pedagogical and moral effort and allo of evangelical life and the spread of devotion to Saint Martin are central.

eight hagiographical books, four of which were dedicentury Gallo-Roman writer. The present work aims to show how the hagiographical work of this learned bishop makes possible the analysis of the historical particularities of an eminently religious society, in
\end{abstract} * El presente estudio forma parte del proyecto de investigación n. ${ }^{\circ}$ 1170661, financiado por el
programa CONICYT-FONDECYT (Chile). 
La figura de Gregorio de Tours es ampliamente conocida desde la perspectiva historiográfica. Gracias a la redacción de su Historia de los francos, él ha sido considerado el principal historiador de la Galia merovingia. Sin embargo, el conjunto de la obra literaria de este obispo de la diócesis turonense -quien sirvió en la sede episcopal de la ciudad de san Martín entre los años 573 y $594^{1}$ - sobrepasa la estricta narración histórica y se puede situar, asimismo, en el ámbito propio de lo que podríamos considerar una hagiografía o teología pastoral, nacida en el seno de las letras latino-cristianas del siglo VI.

Este letrado obispo nació en torno al 538 en el seno de una ilustre familia de la Auvernia -en el corazón de la antigua Galia-, donde recibió una educación clásica que evidenciaba los cambios de las sociedades galorromanas postinvasiones $^{2}$, las cuales se organizaban en torno a las nuevas monarquías germánicas que asumían el ámbito cultural latino. En ese marco histórico que da lugar a una nueva visión conformada por la tradición clásica y los elementos de los pueblos llamados bárbaros, Gregorio redactó un conjunto de cuatro libros que abordan los milagros y las virtudes de san Martín de Tours, llamado De uirtutibus sancti Martini episcopi ${ }^{3}$, el cual fue escrito a lo largo de todo su ejercicio episcopal. En él, nuestro autor se hace heredero de la tradición hagiográfica y episcopal del siglo V, en particular de la biografía de Sulpicio Severo y del poema de Paulino de Périgueux $^{4} \mathrm{y}$, con el celo propio de su labor pastoral, da cuenta de manera novedosa

1 Para una biografía documentada de nuestro autor, véase: Martin HEINZELMANN, Gregory of Tours. History and Society in the Sixth Century, Cambridge, 2001; Martin HeInZELmanN, Gregory of Tours: The Elements of a Biography, en Alexander Callander MURRAY (ed.), A Companion to Gregory of Tours, Leiden-Boston, 2015, pp. 5-34; Luce PIETRI y Marc HeIJMANs, Gregorius Turonensis, en Prosopographie chrétienne du Bas-Empire, t. 4, vol. 1, París, 2013; Luce PIETRI, La ville de Tours du IV au VI siècle: naissance d'une cité chrétienne, Roma, 1983.

2 Bruno DumÉZIL (ed.), Les Barbares, París, 2016.

3 En el mundo hispanohablante esta obra ha sido poco difundida. GREGORIO DE TOURS, De uirtutibus sancti Martini episcopi, ed. por Bruno KRUSCH (Monumenta Germaniae Historica SRM, 1, 2), Hannover, 1969. Las citas del De uirtutibus sancti Martini (en adelante, VM) siguen esta edición latina; la traducción al español es nuestra.

4 El aquitano Sulpicio Severo escribió, en el año 397, un relato biográfico-apologético titulado Vita sancti Martini. Un siglo más tarde, en el 470, Paulino de Périgueux escribió una idealización versificada del santo de Tours. Ambos textos fueron ampliamente conocidos en el ambiente culto de la diócesis turonense. Cfr. Ian WOOD, The Individuality of Gregory of Tours, en Kathleen MrTCHELL y Ian WOOD (eds.), The World of Gregory of Tours, Leiden-Boston, 2002, pp. 34-35; Luce PIETRI, Quand et comment Martin de Tours est-il devenu le saint patron par excellence de la Gaule?, en Jean-Pierre CAILlet et al. (eds.), Des dieux civiques aux saints patrons (IV $V^{e}-V I I^{e}$ siècle), París, 2015, pp. 353-364.

\section{0}


de un nutrido conjunto de milagros que proponen al cristiano de la época un trasfondo teológico de la historia humana ${ }^{5}$, de la pedagogía divina y del testimonio del uir Dei llamado Martín, quien es presentado como un converso ejemplar del siglo IV: monje de Marmoutier, clérigo y discípulo formado en la ortodoxia de Hilario de Poitiers ${ }^{6}$.

En lo que respecta a la estructura de la obra, el libro primero de este tratado expone, a lo largo de cuarenta capítulos, los milagros acontecidos antes de la llegada de Gregorio a la sede turonense. De acuerdo a lo que se puede desprender de su correspondencia con Venancio Fortunato, este texto habría sido terminado en el 576, aproximadamente. En forma paralela, nuestro autor habría trabajado en la recopilación de los milagros de libro segundo, el cual constituye una narración de sesenta capítulos que recogen los sucesos atestiguados por él y ordenados cronológicamente hasta los años 580/581. El libro tercero habría visto la luz entre los años 587 y 588, de acuerdo a las dataciones de los estudios más recientes ${ }^{7}$. Su estructura, similar a la del libro II, se inicia y acaba con un favor recibido por el propio autor y también se organiza en sesenta apartados, distribuidos de manera cronológica. El libro cuarto, a su vez, tiene las particularidades de un texto inconcluso, con cuarenta y siete relatos que, al igual que las otras partes de la obra, incluye un prefacio. También aquí, como ya lo había hecho en los libros segundo y tercero, Gregorio comienza la narración por medio de un milagro de curación hacia su persona.

En el prólogo del primer libro el obispo de Tours presenta, desde una perspectiva que podríamos identificar como hagiográfica, su proyecto histórico-teológico, es decir, la recopilación de los milagros post mortem de san Martín, el apóstol de las Galias, asceta del Loira e instrumento por excelencia del querer

5 Martin Heinzelmann, La réécriture hagiographique dans l'œuvre de Grégoire de Tours, en Beihefte der Francia, 58 (2003), pp. 32-35.

6 Hilario de Poitiers es considerado, a través de su discípulo Martín, como «el primer educador monástico de la Galia romana» (Sulpicio Severo, Vie de saint Martin, t. I, ed. por Jacques FoNTAINE, París, 2004, p. 159). Véase también: Adalbert DE VOGÜÉ, Histoire littéraire du mouvement monastique dans l'Antiquité, tome. IV: Première partie : le monachisme latin, París, 1997, pp. 32-38; Luce PIETRI, La ville de Tours du IV au VI siècle... [ver n. 1], p. VI.

7 En cuanto a la cronología, véase: Richard SHAW, Chronology, Composition, and Authorial Conception in the Miracula, en Alexander Callander MURRAY (ed.), A Companion to Gregory of Tours, Leiden-Boston, 2015, pp. 103-111; Raymond VAN DaM, Saints and Their Miracles in Late Antique Gaul, Princeton, 1993, p. 143; Luce PIETRI y Marc HeIJMANS, Gregorius Turonensis... [ver n. 1], pp. 928-941. 
de Dios entre los francos, en continuidad salvífica desde el Antiguo Testamento hasta sus días ${ }^{8}$ :

nuestro Martín en nada fue inferior a los apóstoles y a los primeros santos, e incluso se puede decir que: feliz hubo de estar Grecia por haber merecido escuchar al apóstol predicador; pero Cristo no abandonó a las Galias pues les concedió el don de poseer a Martín?.

A partir del libro segundo del De uirtutibus el obispo-hagiógrafo escribe con un carácter de testigo y de ministro de fe, y narra un libellus de los milagros que han sucedido por intercesión de san Martín durante su ministerio episcopal, es decir, el historiador se presenta él mismo como garantía de verdad y otorga a su relato un carácter histórico en la medida en que este constituye su voz como pastor que testimonia las mirabilia Dei obradas por intercesión del protector de la diócesis: «así, mis muy queridos, que ninguno de ustedes se permita cuestionarse sobre los misterios divinos, a pesar de que parezca que hemos hablado de una forma rústica» ${ }^{10}$.

Unido a ello, cada acontecimiento portentoso es ubicado bajo coordenadas espaciales y temporales confirmadas por las fiestas religiosas mencionadas a lo largo del texto, aspecto que familiariza al lector de la época con los ritmos litúrgicos de los que formaba parte, especialmente con las dos fechas que rememoraban la gloria de san Martín: 4 de julio y 11 de noviembre ${ }^{11}$. A modo de ejemplo, en el relato de la curación del ciego Leodulfo, ocurrido probablemente en julio del año 593, se señala: «habiendo venido piadosamente a la fiesta del santo obispo, él recobró, al tercer día de esta fiesta, el goce de la luz que había perdido» ${ }^{12}$. A esto se suma el relato en primera persona pues, como ya hemos mencionado, el prelado turonense cuenta algunas de las curaciones y favores recibidos por él mismo.

8 Martin HeinZelmann, La réécriture hagiographique... [ver n. 5], p. 32.

$9 V M, \mathrm{I}, 1$ : sed in nullo inferiorem nostrum potuit repperire Martinum, quem apostolis sanctisque prioribus exaequavit, ita ut etiam diceret: Felicem quidem Graeciam, quae meruit audire apostolum praedicantem; sed nec Gallias a Christo derelictas, quibus donavit habere Martinum.

$10 V M$, II, 1: Unde, dilectissimi, nullus de hoc mysterio, etiamsi rustice videatur dici, disputare praesumat.

11 La fiesta del 4 de julio celebraba el día su ordenación episcopal; aquella del 11 de noviembre, el día de su tránsito. Véase: Martin HeINZELMANN, Une source de base de la littérature hagiographique latine : le recueil de miracles, en Hagiographie, cultures et sociétés. IV -XII siècles. Actes du Colloque organisé à Nanterre et à Paris (2-5 mai 1979), París, 1981, pp. 241-242.

$12 V M, I V, 45:$ ad festivitatem sancti antistitis devotus advenit, cuius post diem tertiam beneficio lucis, quam perdiderat, restauratus est. 
Además de lo anterior, y también vinculado a la labor como obispo de Tours, Gregorio recogió, poco antes de su muerte en el 594, un conjunto de relatos biográficos que se agrupan en el Liber uitae Patrum y el Liber in gloria confessorum ${ }^{13}$, los cuales permiten la consolidación de su proyecto pastoral que deja de manifiesto su deseo de expandir la fe por medio de los exempla uirtutum de diferentes santos, en particular san Martín, propiciando los cultos locales y los patronazgos en toda la región ${ }^{14}$.

\section{EL SENTIDO EDUCADOR DEL MILAGRO}

Cabe señalar, en primer lugar, que más allá de la narración de los acontecimientos y milagros plasmada en el corpus de Gregorio de Tours, somos conscientes de la necesidad de proponer un acercamiento a su persona a partir de coordenadas inherentes a la teología pues, al igual que en toda la literatura cristiana de la época, es posible encontrar en él un evidente plan cristológico que subyace en el conjunto de su obra, y en particular en el texto en cuestión ${ }^{15}$. Sin embargo, y tal como ha sido destacado por el historiador Martin Heinzelmann, el conocimiento teológico de nuestro autor se basa, en gran medida, en un acercamiento hagiográfico que se manifiesta en un plan literario específico, marcado por una estructura que propone modelos tipológicos orientados a la educación de los fieles y a la transmisión de la fe y de la religión, consecuentemente con la labor que él considera propia de su autoridad episcopal ${ }^{16} \mathrm{y}$, asimismo, moral. En relación con esto último, en el prefacio del libro I da cuenta de la intervención de su madre, quien le solicitó que escribiese los milagros que él veía en la cotidianeidad de su ciudad. Así, de un modo retórico y también pedagógico, él recuerda las palabras proferidas por ella: «sería un crimen si guardaras silencio sobre esto» ${ }^{17}$.

13 En la actualidad encontramos una edición crítica de La vida de los padres, publicada por Les Belles Lettres (latín-francés), y una edición latino-inglesa que retoma el texto de Krusch: GREGORIO DE TOURS, La vie des Pères, ed. por Luce PIETRI, París, 2016; GREGORIO DE TOURS, Lives and Miracles, ed. por Giselle DE NIE, Cambridge, MA, 2015.

14 Raymond VAN DAM, Saints and Their Miracles... [ver n. 7], pp. 11-13; Sylvie LABARRE, Le manteau partagé. Deux métamorphoses poétiques de la Vie de saint Martin chez Paulin de Périgueux (Ve s.) et Venance Fortunat (VI s.), París, 1998, pp. 215-216.

15 Cfr. Bruno JUdiC, Introduction. Les avatars de Martin, en ID. et al. (eds.), Un nouveau Martin. Essor et renouveaux de la figure de saint Martin (IV $-X X I^{e}$ siècle), Tours, 2019, p. 11.

16 Martin HeinzelmanN, Gregory of Tours. History and Society... [ver n. 1], p. 153.

$17 V M$, I, praefatio: itaque ne dubites et haec agere non desistas, quia crimen tibi erit, si ea tacueris. 
En su rol de obispo, Gregorio comprende la importancia del papel de la educación cristiana y de una pastoral educativa ${ }^{18}$ transmitida a través de los relatos portentosos y, a pesar del recurso literario utilizado para excusarse de su rusticidad gramatical plasmada en su escritura, deja en claro la importancia de la figura de san Martín y da cuenta del objetivo de su esfuerzo narrativo. $\mathrm{Al}$ respecto escribe: «así pues, tengo la confianza que, si mi estilo inculto no puede embellecer este libro, al menos los altos y luminosos hechos del glorioso pontífice lo pondrán a la luz» ${ }^{19}$. En este sentido, y de acuerdo con lo que ha sido notado para el caso de la Historia de los francos, Gregorio buscaba ampliar el círculo de creyentes a los que se dirigía el texto, es decir, deseaba promover el modelo martiniano para todos los fieles cristianos, tanto para los más doctos como para los más rustici ${ }^{20}$. Ante esto aparece, por tanto, la idea de una ecclesia Dei que subyace en todo el texto de nuestro autor galorromano, y que invita a una lectura de la realidad en clave interpretativa también teológica.

La labor episcopal de Gregorio se asienta en una continuidad literaria con la tradición clásica, más concretamente con la idea de la aedificatio por medio de los relatos de varones ilustres y de vidas de santos ${ }^{21}$. Esta edificación ejemplar, siempre asociada al conocimiento de un hecho hagiográfico, va unida al deseo de un perfeccionamiento moral y espiritual ${ }^{22}$, y supone un objetivo didáctico-pastoral que se asienta en la concepción escatológica intrínseca que es parte del cristianismo, y que constituye todo el marco de referencia de la obra del turonense, tal como lo expresa él mismo en el prólogo del Libro de la vida de los Padres: «la vida de ellos serviría para la edificación de la Iglesia» ${ }^{23}$.

18 Cfr. J. SCHLICK, Composition et chronologie des De virtutibus sancti Martini de Grégoire de Tours, en Studia Patristica, VII (1966), p. 281.

$19 V M$, I, praefatio: confidimus ergo orantibus vobis, quia, etsi non potest paginam sermo incultus ornare, faciet eam gloriosus antistis praeclaris virtutibus elucere.

20 Martin Heinzelmann, Gregory of Tours. History and Society... [ver n. 1], pp. 158-159.

$21 \mathrm{Al}$ respecto se puede encontrar una literatura relevante de la cual mencionamos: Brigitte BEAUJARD, Le culte des saints en Gaule. Les premiers temps. D'Hilaire de Poitiers à la fin du VI siècle, París, 2000; Martin HeInZELmanN, Studia sanctorum. Éducation, milieux d'instruction et valeurs éducatives dans l'hagiographie en Gaule jusqu'à la fin de l'époque mérovingienne, en Michel SoT (ed.), Haut Moyen-Âge. Culture, éducation et société. Études offertes à Pierre Riché, Nanterre, 1990, pp. 105-138; Aline Rousselle, Croire et guérir. La foi en Gaule dans l'Antiquité tardive, París, 1990.

22 Martin HeInZELMANN, Grégoire de Tours et l'hagiographie mérovingienne, en Antonella DEGL'INNOCENTI et al. (eds.), Gregorio Magno e l'agiografia fra IV e VII secolo, Florencia, 2007, p. 155. Véase también: Sylvie LABARRE, La figure de saint Martin chez Grégoire de Tours, en Bruno JUDIC et al. (eds.), Un nouveau Martin..., op. cit., pp. 214-215.

23 GREGORIO DE TOURS, Liber uitae Patrum, praefatio: quorumque uitae tramitem certis relationibus cognitum eclesiam aedificare putaui. 
De este modo, el conjunto de milagros de san Martín no vendría a ser una mera compilación o un ramillete de acontecimientos maravillosos sin un orden interno y sin una lógica ordenadora sino, por el contrario, ellos constituyen una parte fundamental de un proyecto presentado como integral y coherente, transversalmente expresado en la totalidad de las obras, con san Martín como modelo de conversión, tal como ha sido propuesto por Heinzelmann ${ }^{24}$. Es a partir de esta premisa que podemos identificar y analizar algunos elementos propios del programa pastoral del obispo Gregorio, así como algunos conceptos fundamentales para una mejor aproximación a la visión histórico-providencialista de este escritor latino-cristiano de la segunda mitad del siglo VI.

El auditorio al cual nuestro autor dirige la obra no corresponde a un grupo de hombres letrados capaces de leer los grandes tratados sobre la vida del apóstol de las Galias, es decir, aquellos escritos por el retórico Sulpicio Severo o por los poetas Paulino de Périgueux o el propio Venancio Fortunato, este último contemporáneo y amigo de Gregorio. El texto se dirige, más bien, al fiel que no ha sido formado en las grandes letras y que por medio de la vivencia ejemplarizadora del milagro se siente invitado al seguimiento de las enseñanzas evangélicas transmitidas por el santo protector. Allí radicaría, en cierta forma, un primer valor que se presenta de modo explícito a lo largo de la narración sobre san Martín, es decir, los milagros en sí mismos no son sólo una recopilación de hechos fantásticos de fácil comprensión, sino que constituyen per se una introducción sensible al sentido cristológico del tiempo y de la historia humana ${ }^{25}$. En otras palabras, los sucesos portentosos constituyen un recuerdo constante de que Dios es quien realiza las maravillas en el mundo, por su voluntad omnipotente y por medio de los hombres de probada santidad, en particular el obispo-monje Martín ${ }^{26}$. A modo de ejemplo, en el libro primero

24 Martin Heinzelmann, La réécriture hagiographique... [ver n. 5], p. 22.

25 Los milagros señalados por Gregorio de Tours se agrupan en diferentes categorías. Las más representativas son, a nuestro juicio: a) conversión de nobles y de hombres sencillos, b) curación de enajenados, c) curación de enfermos (disentería, fiebres y otras enfermedades), d) liberación de poseídos, f) curación de lisiados (ciegos, mudos, paralíticos, tullidos), g) resurrección de muertos, h) curaciones de animales, i) liberación de prisioneros, j) multiplicaciones, k) manifestaciones luminosas y portentos naturales. Para una aproximación a los milagros en el conjunto de la obra de nuestro autor, véase: Joan M. PETERSEN, The Dialogues of Gregory the Great in Their Late Antique Cultural Background, Toronto, 1984, p. 125.

26 Guy PHILIPPART, L’hagiographie, histoire sainte des «amis de Dieu», en Guy PHILIPPART (ed.), Hagiographies. Histoire internationale de la littérature hagiographique latine et vernaculaire en Occident des origines à 1550, t. IV, Turnhout, 2006, p. 25; Brigitte BEAUJARD, Le culte des saints en Gaule... [ver n. 21], p. 272. 
del De uirtutibus el propio autor recuerda los consejos de su madre, los cuales resultan particularmente ilustradores al este respecto: «¿ignoras que aquel que nos habla un lenguaje adaptado a la inteligencia del pueblo, como tú puedes hacerlo, es por esto mismo mejor comprendido? ${ }^{27}$. Más adelante, en el libro segundo, añade: «ante la majestad de Dios vale más la simplicidad pura que la argucia de los filósofos» ${ }^{28}$.

El obispo Gregorio, de acuerdo con los cánones retóricos de la literatura hagiográfica, enmarca su proyecto pedagógico-pastoral en dos aspectos que resultan fundamentales para entender el sentido de esta obra: a) en primer lugar él mismo se hace eco de la tradición oral y pone por escrito aquello que ha escuchado en su diócesis y, b) él se transforma en testigo de los acontecimientos de sus días y da fe de ellos, en tanto que fiel devoto de san Martín y obispo de la diócesis turonense. Esto último tendrá una relevancia particular ya que será él -la cabeza de la iglesia de Tours- quien narrará las gestas del santo patrono y quien, por medio de su labor literaria y de su celo apostólico, se transformará, hasta cierto punto, en uno más de los milagros del santo prelado al poder describir el conjunto de los portentos obrados por Cristo a través del asceta del Loira.

Por lo que atañe al proyecto pastoral del obispo galorromano, consideramos que este se encuentra íntimamente unido a una epifanía de la Iglesia celeste, la cual se manifiesta por medio de los milagros post mortem, que son aquellos que plasman el devenir histórico y que lo impregnan, de cierta manera, del sentido cristológico de la historia humana en tanto que bistoria salutis. Los milagros tienen una significación interior que, tal como expresa Marc van Uytfanghe, constituye un exemplum del camino interior del creyente por el cual este debe necesariamente progresar en el descubrimiento de Dios, en la cotidianeidad de la vida ${ }^{29}$.

Sin ánimo de ser reiterativos, hemos de señalar que el proyecto catequético de Gregorio fija su atención, principalmente, en la idea de la aedificatio ecclesiae $y$, en particular, en las figuras episcopales y en la trascendencia de sus obras pues

$27 V M$, I, praefatio: Et nescis, quia nobiscum propter intellegentiam populorum magis, sicut tu loqui potens es, habetur praeclarum?

$28 V M$, II, 1: quia apud Dei maiestatem magis simplicitas pura quam philosophorum valet argutia.

29 Véase Marc VAN UYTFANGHE, La controverse biblique et patristique autour du miracle, et ses répercussions sur l'hagiographie dans l'Antiquité tardive et le haut Moyen Age latin, en Hagiographie, cultures et sociétés. IVe-XII siècles. Actes du Colloque organisé à Nanterre et à Paris (2-5 mai 1979), París, 1981, p. 219. 
el éxito del plan divino en la historia se vería reflejado en estos hombres que recrean de manera ejemplar el mensaje de Cristo. Así, y tal como lo ha expresado Heinzelmann, el milagro operado por el santo -en este caso san Martín-, permite reconocer la labor de un «agente de la uirtus Dei» ${ }^{30}$, es decir, de un confesor de la fe y de un testigo fiel, tal como el mártir lo fue durante los primeros siglos del cristianismo $^{31}$.

Retomando el hilo propio del proyecto hagiográfico de Gregorio, no está de más la incorporación del concepto que, para referirse a estos asuntos, puede denominarse «una pastoral educativa ${ }^{32}$, la cual representa una verdadera educación cristiana basada en el ejemplo y en el papel pedagógico de los milagros. Así, al hablar del santo, de la reliquia y del milagro entendemos una tríada que forma el tríptico que ilustra la fisonomía del creyente de la época, quien por medio de la devoción al patrono-protector se siente vinculado con el mensaje cristiano. Por ello, y de un modo ejemplar, el milagro adquiere una dimensión personal pero también, y como condición necesaria, una dimensión social y comunitaria, la cual se ve reflejada en el deseo explícito del letrado turonense de continuar con la labor comenzada por su predecesor, el obispo Perpetuo, a quien identifica como discípulo del bienaventurado Martín ${ }^{33}$.

De este modo él prolonga la tradición de su diócesis y entrega una suerte de herencia espiritual a los cristianos de su región ${ }^{34}$, pues su De uirtutibus sancti Martini episcopi no hace otra cosa más que predicar la manera en la cual, providencialmente, san Martín «fue destinado por Dios para la salvación de las Galias» ${ }^{35}$. En este aspecto radicaría, entonces, lo que nuestro obispo ha entendido como una de sus labores primordiales, en concreto, la conservación de la memoria y la difusión de los milagros post mortem acaecidos a los pies de la tumba del santo varón, labor que no fue tratada ni por Sulpicio el biógrafo ni por Paulino el poeta ${ }^{36}$.

30 Martin HeInZElmann, Pouvoir et idéologie dans l'hagiographie mérovingienne, en Edina BOZÓKY (ed.), Hagiographie, idéologie et politique au Moyen Âge en Occident. Actes du colloque international du Centre d'Études supérieures de Civilisation médiévale de Poitiers. 11-14 septembre 2008, Turnhout, 2012, p. 50.

31 Brigitte BEAUJARD, Le culte des saints en Gaule... [ver n. 21], pp. 54-58; G. PHILIPPART, L'bagiographie, histoire sainte... [ver n. 26], p. 31.

32 J. SCHLICK, Composition et chronologie... [ver n. 18], p. 281.

$33 V M, \mathrm{I}, 2$ : sanctus vero Perpetuus episcopus, merito beati discipulus praedicandus.

34 Para el desarrollo del culto a san Martín véase: R. VAN DAM, Saints and Their Miracles... [ver n. 7], pp. 13-28.

$35 V M, \mathrm{I}, 3:$ ad salvationem Galliarum opitulante Deo diregitur.

36 Cfr. $V M$, I, 3: quia hoc erit scribendi studium, quod in illo Severi aut Paulini opere non invenitur insertum. 


\section{EL EJEMPLO DE SAN MARTÍN Y LA CONVERSIÓN DEL CREYENTE}

Ya hemos hablado del deseo de edificación y de fortalecimiento de la Iglesia que subyace en la obra hagiográfica del obispo de Tours. A pesar de ello, no hemos insistido lo suficiente en la idea de comunidad que se dejaría vislumbrar a través de la sencilla enumeración y narración de acontecimientos portentosos. El relato de los milagros sitúa al lector en un orden eclesial significativo pues el fiel laico $^{37}$, la ecclesia Dei en tanto que cuerpo místico, se manifiesta de manera directa por medio de la alabanza a Dios y del elogio a los santos. Por lo tanto, el acontecimiento evidentemente sobrenatural -o sea, el milagro- adquiere un sentido anagógico que permite al lector familiarizado con las Escrituras un segundo nivel de lectura, necesariamente teológico y, por extensión, cristocéntrico.

Supuesto lo anterior, al retomar la significación y el sentido del milagro en cuanto manifestación divina, es posible afirmar que los acontecimientos sucedidos por la acción de Dios a través de la súplica de san Martín no serían una mera coincidencia o resultados del azar. Estos cumplirían, más bien, una labor asociada a una epifanía de la historia, la cual soporta y recrea una realidad escatológica que tendría una presencia mística en el mundo temporal, en el devenir de los fieles cristianos de aquellos días. A modo de ejemplo de lo recién expuesto, podemos mencionar cómo la historia de la Iglesia gala, representada de manera particular en la diócesis turonense, muestra a Cristo en la figura de los obispos y, de modo preclaro, en la persona del prelado Martín. Por ello, el proyecto sistemático que se presenta claramente desde el libro I comprende la función tutora del santo, así como la protección paternal en el orden del cuerpo místico antes mencionado, por medio de la administración de las múltiples gracias divinas derramadas en el grupo de fieles que se desplazan y acuden fervorosamente, de modo personal y comunitario, al santuario del Loira (basílica) para pedir la intercesión del monjeobispo durante la misa dominical o una festividad litúrgica ${ }^{38}$.

El milagro, por lo tanto, refuerza la fe de los creyentes y ayuda a vivir la fidelidad al bautismo ${ }^{39}$, pues él atestigua el favor divino de un modo físico y, asi-

37 El esplendor del culto a san Martín abarcaba a todos los miembros de la sociedad gala, tal como lo señala el panorámico estudio de Luce PIETRI, Quand et comment Martin de Tours est-il devenu le saint patron par excellence de la Gaule?... [ver n. 4], p. 359.

38 Cfr. Sylvie LABARRE, Les saints guérisseurs et protecteurs chez Grégoire de Tours et Venance Fortunat, en Jean-Pierre Caillet et al. (eds.), Des dieux civiques aux saints patrons (IV ${ }^{e}-V I I^{e}$ siècle), París, 2015, pp. 317-318.

39 Guy-Marie OuRY, Le miracle dans Grégoire de Tours, en Histoire des miracles. Actes de la Sixième Rencontre d'Histoire Religieuse tenue à Fontevraud les 8 et 9 octobre 1982, Angers, 1983, p. 24. 
mismo, se constituye en una suerte de admonición testimonial, según cada caso, la que se hace presente por medio de la palabra del prelado historiador, Gregorio, quien se muestra como el garante de aquello que narra con detalle y que a la vez advierte y refuerza.

El conjunto de testimonios de los diferentes milagros debe entenderse, al mismo tiempo, bajo el prisma del juicio definitivo, lo cual da la idea de una iglesia escatológica que se presenta de manera equivalente por medio del obispo protector, un alter Christus. En esta línea apunta, por ejemplo, el relato de la muerte de san Martín y su sentido cristológico:

no es algo de poca importancia el remarcar que Dios lo haya recibido en su paraíso el día preciso en el cual el mismo Señor y Redentor, victorioso desde los infiernos, fue resucitado, y que aquel quien siempre había celebrado sin mancha alguna las solemnidades del domingo, haya sido recibido en el reposo después de los tormentos de la vida, en domingo ${ }^{40}$.

La muerte de san Martín habría tenido una gran significación para el obispo hagiógrafo, quien ha dotado a este acontecimiento de un connotación teológicohistórica que ha sido reconocida por Luce Pietri, entre otros ${ }^{41}$. El deceso del santo protector vendría a significar el umbral de una época pues, a partir de ese momento, sería posible hablar de «los tiempos de Martín ${ }^{42}$ no sólo bajo una concepción cronológica sino, asimismo, sobrenatural. Dicha afirmación se correspondería, en definitiva, con la idea de san Martín como el modelo más perfecto de discipulado de Cristo, y su actividad terrestre serviría de ejemplo para los cristianos quienes, favorecidos por los milagros de la basílica turonense, adoptarían un comportamiento penitente de ayuno y oraciones ${ }^{43}$ para luego, consecuentemente, aceptar un cambio de vida y una conversión del alma. Por esto la oración privada y la penitencia son el denominador común de los cuatro libros de los milagros de san Martín pues el cuerpo, en tanto que templo, se debe preparar por medio de la purificación interior y del reconocimiento del bien superior para recibir el favor divino. En otras pala-

$40 V M$, I, 3: Quod non parvi meriti fuisse censetur, ut illa die eum Dominus in paradiso susciperet, qua idem Redemptor et Dominus victor ab inferis surrexisset; et, ut qui dominica solemnia semper celebraverat inpollutae, post mundi pressuras dominica die locaretur in requie.

41 Luce PIETRI, La succession des premiers évêques tourangeaux : essai sur la chronologie de Grégoire de Tours, en Mélanges de l'École française de Rome, 94/2 (1982), pp. 580-581; Martin HEINZELMANN, Structures typologiques de l'histoire d'après les histoires de Grégoire de Tours, en Recherches de Science Religieuse, 92/4 (2004), p. 573.

42 Martin HeinzelmanN, Structures..., op. cit.

43 Para ver las actividades de los penitentes se puede revisar, a modo de ejemplo: $V M$, I, 19, 21. 
bras, las curaciones no se completan hasta que el enfermo corporal no se entrega a la oración penitente para salvar, en primer lugar, su alma ${ }^{44}$ :

damos gracias a Dios Todopoderoso que se dignó concedernos un médico para sanar nuestras debilidades, para cicatrizar nuestras heridas y para darnos medicinas eficaces [...]. Cuando corren las lágrimas y se sucede una verdadera compunción, desde el fondo del corazón brotan los lamentos y nos golpeamos nuestros pechos culpables; entonces del llanto nace la alegría, de la falta nace el perdón, y así el corazón dolorido encuentra su remedio ${ }^{45}$.

Por consiguiente, la vida y testimonio del santo obispo Martín supondría una bendición divina asociada a una pedagogía de la salvación que inauguraría una nueva época de la Iglesia de Cristo ${ }^{46}$, marcada por las sanaciones que no sólo restablecerían la salud de los miembros físicos, sino que, más importante aún, «limpiarían las manchas mismas de las conciencias» ${ }^{47}$ para alcanzar la salvación de las almas de un pueblo en su conjunto. En consecuencia, y desde una óptica teológica, la narración hagiográfica sobre las virtudes de san Martín escrita por Gregorio responde a un contexto específico en el cual los signos divinos, manifestados por los santos patronos y sus obras, aparecen como signos vivientes de Cristo, Señor de la historia humana ${ }^{48}$. El enfermo, consciente de su pecado que se manifiesta en el padecimiento de la carne, obtiene la purificación y asimismo la reincorporación en el seno de la comunidad cristiana a la cual pertenece ${ }^{49}$, tal como lo manifiesta el milagro de curación de la mano de un campesino que no observó el reposo dominical:

cuatro años después él vino a la basílica del santo; hizo oración y fue curado. Entonces, él proclamó delante de todo el pueblo que nadie debía imitar lo que él había hecho, y que un agricultor avaricioso no debía profanar la solemnidad

44 Para la dinámica pecado-enfermedad y curación-salvación, véase: J. K. KITCHEN, Gregory of Tours, Hagiography, and the Cult of the Saints in the Sixth Century, en Alexander Callander MURRAY (ed.), A Companion to Gregory of Tours, Leiden-Boston, 2015, pp. 398-402.

$45 V M$, III, praefatio: gratias agimus omnipotenti Deo, qui nobis talem medicum tribuere dignatus est, qui infirmitates nostras purgaret, vulnera dilueret, ac salubria medicamenta conferret [...]. Si defluant lacrimae, et conpunctio vera succedat, si ab imo corde mittantur suspiria, et pectora facinorosa tundantur, invenit ploratus laetitiam, culpa veniam, dolor pectoris pervenit ad medellam.

46 Martin HeinzelmanN, Structures typologiques... [ver n. 41], p. 584.

47 Cfr. VM, III, 60: ipsas illas conscientiarum maculas abstergit ac levigat.

48 Un santo protector contemporáneo a san Martín es, por ejemplo, Victricio de Ruan. Para el caso itálico san Benito resulta particularmente importante. Martin HeINZELMANN, Structures typologiques... [ver n. 41], p. 592.

49 Para una interpretación de la enfermedad en la visión de Gregorio véase: Sylvie LABARRE, Les saints guérisseurs et protecteurs chez Grégoire de Tours... [ver n. 38], pp. 319-322. 
del domingo, y que la humanidad dañada no debía corromper, por los trabajos de este mundo, el misterio celeste de la santa Resurrección y de nuestra redención ${ }^{50}$.

En efecto, el proyecto literario-pastoral de Gregorio tendría un sentido unitario, con un objetivo evangelizador y con resultados distintos de acuerdo con la naturaleza histórica o hagiográfica de sus obras. Este proyecto va unido a una tradición espiritual que él concibe en tanto que obispo de Tours, y que él mismo lega a la posteridad como modo de alabanza a Dios por medio del elogio al monje-obispo de su diócesis quien, gracias a la virtud de la humildad, «nunca se alabó a sí mismo» ${ }^{51}$. Frente a este panorama, Cristo es el fundamento tipológico de su De uirtutibus sancti Martini y las distintas dimensiones del santo del Loira se pueden resumir bajo un único rol atribuido a él de manera relevante, universal e indefectible, tal como aparece en el libro IV: «Martín es patrono especial de todo el orbe» ${ }^{52}$.

Dentro del ámbito de la iglesia del siglo VI, san Martín es presentado como el patrono de los pueblos piadosos, de los cristianos devotos que siguen el ejemplo de una vida gloriosa ${ }^{53}$, hasta cierto punto inimitable. Efectivamente, es el ejemplo de virtud que nuestro autor identifica como una característica inherente al amicus Dei-Martín- ${ }^{54}$, es decir, un creyente que ha confesado la fe y que ha testimoniado hasta el final la doctrina de los Evangelios ${ }^{55}$ y que, por medio de los milagros dispensados desde su tumba, da testimonio de las cosas santas, sigue predicando después de la muerte ${ }^{56}$, y puede transformar los espíritus estériles para la edificación de la Iglesia de Cristo.

La labor del cristiano es concebida, asimismo, como una tarea de propagación de la fe. El hombre sanado se encuentra, ciertamente, liberado del mal y se convier-

$50 V M$, III, 29: Post quattuor vero annos ad basilicam sancti advenit; oratione quoque facta, sanatus est, praedicans populis, ne factum quis meditaretur, ne tanti die solemnia avarus agricola macularet, ne redemptionis sacrae resumptionis nostrae caeleste mysterium humanitas infirma, terrena exercens opera, dissolveret.

$51 V M, \mathrm{I}, 5$ : cuius laus ab eius ore numquam recessit.

$52 \mathrm{VM}, \mathrm{IV}$, praefatio: toto orbi peculiare patrono Martino antistite.

53 Cfr. $V M$, I, 14: quod ope sancti Martini recto iudicio reputat, qui vicinitate sua sibi devotis populis sedulam exhibuit praesentemque custodiam.

$54 V M$, IV, praefatio: ut decet amicum Dei.

55 Guy PHILIPPART, L'hagiographie, bistoire sainte... [ver n. 26], p. 31.

56 Cfr. $V M$, II, 40: vi enim me inpellit amor patroni, et quia adhuc esse eum denuntiavi praedicatorem, dicam, quid contigerit nuper. Véase, también: Martin HEINZELMANN, Grégoire de Tours et l'hagiographie... [ver n. 22], p. 181. 
te en testigo elocuente para los otros. El fiel curado sirve de modelo de conversión para sus contemporáneos y, sirviéndose de los favores recibidos, testimonia la virtud del santo, la cual constituye en sí misma una sabiduría de vida que se asienta en la acción de Dios. El santo, en este caso el obispo-asceta del Loira, se transforma en una suerte de educador de la fidelidad a Cristo y del seguimiento a su doctrina, y sus milagros son la demostración fidedigna de la fuerza de la Iglesia de sus días y del cumplimiento de las Escrituras. Por medio de la acción de san Martín, comenzada en vida desde el siglo IV y continuada históricamente por medio de los testimonios hasta los días de Gregorio, el plan divino se presenta bajo las coordenadas espaciotemporales, como un diseño único en el cual la intervención providente de Dios y de sus milagros coronan la evolución de una comunidad cristiana y de una diócesis en particular, tal como ha sido notado de manera particular para la Historia de los francos y de forma extensiva para el De uirtutibus ${ }^{57}$.

Pocas veces el exemplum funcionaría con tanta eficacia como en el caso de la narración de los milagros del obispo Martín. En dichos acontecimientos milagrosos post mortem se enfatizan las gestas providenciales dispensadas por «los vasallos de Dios» ${ }^{58}$, en este caso el santo patrono. El marco elegido por el autor, a nuestro juicio, se centra en la confesión de la fe y en la lucha contra el pecado, lo que se deja en evidencia a lo largo de los cuatro libros que conforman el $D e$ uirtutibus, por medio de un criterio basado en la práctica de las virtudes cristianas: la caridad, la castidad, la humildad y la paciencia. Si bien este rasgo resulta común a los relatos hagiográficos anteriores -Sulpicio Severo y Paulino de Périgueux-, también se constituye en el hilo conductor del modelo tipológico de Cristo, en donde la descripción del milagro y de su contexto se transforma también en una especie de instrumento histórico del Señor y en un medio de sanación y de prueba de fidelidad a la manera del asceta del Loira, a quien Gregorio llama mártir pues padeció toda clase de obstáculos y de agresiones, conservando siempre intacta la gloria del cuerpo y del espíritu: «supo guardar intacta la gloria de la virginidad, soportó triunfalmente el martirio, resistiendo ya fuesen las trampas secretas como las ofensas públicas ${ }^{59}$.

57 Cfr. Luce PIETRI, La ville de Tours du IV au VI siècle... [ver n. 1], pp. XIII, 321; Martin HeINZELMANN, Structures typologiques... [ver n. 41], p. 594.

58 En cuanto al tema del milagro post mortem, consúltese: Joan M. PETERSEN, The Dialogues of Gregory the Great in Their Late Antique Cultural Background... [ver n. 25]; Guy PHILIPPART, L'bagiographie, histoire sainte... [ver n. 26], p. 25.

$59 V M$, II, 60: scilicet ut beatus Martinus, qui viduatus ab hoc mundo virginitetis custodivit integrum decus, martyrium etiam vel in occultis insidiis vel in publicis iniuriis triumphabiliter adimplevit. 
De hecho, para el obispo-historiador los milagros martinianos son una parte de la realidad cotidiana que le ha tocado vivir, una expresión de la importancia de los santuarios y una consecuencia natural de la devoción a las reliquias conservadas en sus días ${ }^{60}$. En este sentido, su cosmovisión general le otorgaría una clara idea de una epifanía en la historia, pues el paradigma de la fe tendría para él una correspondencia directa en los signos vivientes de la intervención de Dios y, más claro aún, de Cristo como Señor de la historia. En sus propias palabras, afirma:

nuestra fe nos dice que un solo Señor interviene por la virtud de un gran número de santos, quienes no son divididos a causa del poder pues son considerados iguales en el cielo e idénticos, en cuanto a los milagros, sobre la tierra ${ }^{61}$.

En definitiva, estaríamos frente a una tradición patrística transmitida por medio de los cánones hagiográficos, en donde los trazos de los grandes autores latino-cristianos se manifiestan con la misma familiaridad que los misterios teológicos de la religión y de la Providencia divina en la historia ${ }^{62}$.

\section{A MODO DE CONCLUSIÓN}

Para finalizar, al enfilar el último tramo de este trabajo, cabe destacar que tras la estructura narrativa de los cuatro libros de las virtudes de san Martín es posible vislumbrar un hipotexto que se relaciona directamente con el aspecto tipológico que hemos mencionado con anterioridad, es decir, Cristo es el verdadero autor de cada milagro y el fiel debe saberlo y recordarlo. Este modelo literario por excelencia representa, también, un ideal eclesial y remite a un trasfondo teológico que ha sido poco remarcado, tal como se ha afirmado más arriba. El aspecto filológico-literario no constituye el único o mejor punto de análisis de la obra de Gregorio, pues el trasfondo hagiográfico excede ampliamente el estricto

60 Giuseppe CREMASCOLI, Il miracolo nell'agiografia di Gregorio Magno e di Gregorio di Tours, en Antonella DEGL'INNOCENTI et al. (eds.), Gregorio Magno e l'agiografia fra IV e VII secolo, Florencia, 2007, pp. 252-255. Una panorámica del culto a las reliquias se encuentra en Aline RoUSSELLE, Croire et guérir. La foi en Gaule... [ver n. 21], pp. 109-122.

$61 V M, I V, 12$ : Verumtamen fides nostra retenet, in multorum sanctorum virtutibus unum Dominum operari, et nec illos disiunctos virtutibus, quos caelo pares, miraculis Dominus aequales reddit in terris.

62 Martin Heinzelmann, The Works of Gregory of Tours and Patristic Tradition, en Alexander Callander MURRAY (ed.), A Companion to Gregory of Tours, Leiden-Boston, 2015, pp. 299-305; Martin HeINZELMANN, Grégoire de Tours et l'hagiographie... [ver n. 22], p. 192. 
orden estético o artístico. Es más, la belleza de sus letras sólo estaría dada por el mismo ideal cristocéntrico que identifica las formulaciones de cada acontecimiento maravilloso, y su tarea consistiría en engrandecer las filas de la Iglesia de Tours para mayor gloria de Dios ${ }^{63}$.

En suma, la confesión de la fe se presenta como un eje programático y, a pesar de la narración de milagros aparentemente desproporcionada, los libros sobre las virtudes del santo monje-obispo turonense se enmarcan en una literatura apologética ejemplar, nutrida del mosaico de acontecimientos obrados en el santuario del Loira y concretados en manifestaciones sensibles, es decir, en las mirabilia Dei. En conclusión, esta recopilación martiniana llevada a cabo por el obispo Gregorio de Tours viene a ser un fiel reflejo de una fisonomía cultural en la cual el evento prodigioso se concibe como verídico, como punto de contacto cierto con el mundo sobrenatural en el que se inserta el cuerpo místico del cual él y su Iglesia merovingia forman parte.

\section{REFERENCIAS BIBLIOGRÁFICAS}

\section{Fuentes editadas}

GREGORIO DE TOURS, De uirtutibus sancti Martini episcopi, ed. por Bruno KRUSCH (Monumenta Germaniae Historica SRM, 1, 2), Hannover, 1969.

GREgorio De TOURS, La vie des Pères, ed. por Luce Pietri, París, 2016.

GREGORIO DE TOURS, Le livre des miracles de saint Martin, ed. por Henri BORDIER, ClermontFerrand, 2007.

GREGORIO DE TOURS, Lives and Miracles, ed. por Giselle DE NIE, Cambridge, MA, 2015. PAUlino de PÉRIgUeUX, Vie de saint Martin, t. I, ed. por Sylvie LabarRe, París, 2016. SULPICIO SEVERO, Vie de saint Martin, t. I, ed. por Jacques FonTAINE, París, 2004 venancio fortunato, Vie de saint Martin, ed. por Solange QueSnel, París, 1996.

\section{Estudios}

BEAUJARD, Brigitte, Le culte des saints en Gaule. Les premiers temps. D'Hilaire de Poitiers à la fin du VI siècle, París, 2000.

63 Cfr. Lellia CRACCO RUGGINI, Il miracolo nella cultura del tardo impero: concetto e funzione, en Hagiographie, cultures et sociétés. IV $V^{e}$ XII siècles. Actes du Colloque organicé à Nanterre et à Paris (2-5 mai 1979), París, 1981, pp. 170-171; Martin HeINZELMANN, La réécriture bagiographique... [ver n. 5], p. 37. 


\section{GREGORIO DE TOURS Y LA HAGIOGRAFÍA DEL SIGLO VI}

CRACCO RUGGINI, Lellia, Il miracolo nella cultura del tardo impero: concetto e funzione, en Hagiographie, cultures et sociétés. $I V^{2}-X I I^{e}$ siècles. Actes du Colloque organicé à Nanterre et à Paris (2-5 mai 1979), París, 1981.

CREMASCOLI, Giuseppe, Il miracolo nell'agiografia di Gregorio Magno e di Gregorio di Tours, en Antonella DEGL'INNOCENTI et al. (eds.), Gregorio Magno e l'agiografia fra IV e VII secolo, Florencia, 2007.

DAM, Raymond van, Saints and Their Miracles in Late Antique Gaul, Princeton, 1993.

DumÉZIL, Bruno (ed.), Les Barbares, París, 2016.

HEINZELMANN, Martin, Une source de base de la littérature hagiographique latine: le recueil de miracles, en Hagiographie, cultures et sociétés. IV $V^{e} X I I^{e}$ siècles. Actes du Colloque organisé à Nanterre et à Paris (2-5 mai 1979), París, 1981.

- Studia sanctorum. Éducation, milieux d'instruction et valeurs éducatives dans l'bagiographie en Gaule jusqu'à la fin de l'époque mérovingienne, en Michel SoT (ed.), Haut Moyen-Âge. Culture, éducation et société. Études offertes à Pierre Riché, Nanterre, 1990.

- Gregory of Tours. History and Society in the Sixth Century, Cambridge, 2001.

- La réécriture hagiographique dans l'œuvre de Grégoire de Tours, en Beibefte der Francia, 58 (2003).

- Structures typologiques de l'bistoire d'après les histoires de Grégoire de Tours, en Recherches de Science Religieuse, 92/4 (2004).

- Grégoire de Tours et l'hagiographie mérovingienne, en Antonella DEGL'INNOCENTI et al. (eds.), Gregorio Magno e l'agiografia fra IV e VII secolo, Florencia, 2007.

- Pouvoir et idéologie dans l'hagiographie mérovingienne, en Edina BOZÓKY (ed.), Hagiographie, idéologie et politique au Moyen Âge en Occident. Actes du colloque international du Centre d'Études supérieures de Civilisation médiévale de Poitiers. 11-14 septembre 2008, Turnhout, 2012.

- The Works of Gregory of Tours and Patristic Tradition, en Alexander Callander MURRAY (ed.), A Companion to Gregory of Tours, Leiden-Boston, 2015.

- Gregory of Tours: The Elements of a Biography, en Alexander Callander MURRAY (ed.), $A$ Companion to Gregory of Tours, Leiden-Boston, 2015.

JUDIC, Bruno, Introduction. Les avatars de Martin, en ID. et al. (eds.), Un nouveau Martin. Essor et renouveaux de la figure de saint Martin (IVe-XXIe siècle), Tours, 2019.

Kitchen, J. K., Gregory of Tours, Hagiography, and the Cult of the Saints in the Sixth Century, en Alexander Callander MURRAY (ed.), A Companion to Gregory of Tours, Leiden-Boston, 2015.

LABARRE, Sylvie, Le manteau partagé. Deux métamorphoses poétiques de la Vie de saint Martin chez Paulin de Périgueux (Ve s.) et Venance Fortunat (VIe s.), París, 1998.

- Les saints guérisseurs et protecteurs chez Grégoire de Tours et Venance Fortunat, en Jean-Pierre CAILlET et al. (eds.), Des dieux civiques aux saints patrons (IV -VII siècle), París, 2015.

- La figure de saint Martin chez Grégoire de Tours, en Bruno JUDIC et al. (eds.), Un nouveau Martin. Essor et renouveaux de la figure de saint Martin (IV ${ }^{2}-X X I^{e}$ siècle), Tours, 2019.

OuRY, Guy-Marie, Le miracle dans Grégoire de Tours, en Histoire des miracles. Actes de la Sixième Rencontre d'Histoire Religieuse tenue à Fontevraud les 8 et 9 octobre 1982, Angers, 1983.

Petersen, Joan M., The Dialogues of Gregory the Great in Their Late Antique Cultural Background, Toronto, 1984. 


\section{MARCELO AGUIRRE DURÁN}

PHILIPPART, Guy, L’hagiographie, histoire sainte des «amis de Dieu», en Guy PHILIPPART (ed.), Hagiographies. Histoire internationale de la littérature hagiographique latine et vernaculaire en Occident des origines à 1550, t. IV, Turnhout, 2006.

PIETRI, Luce, La succession des premiers évêques tourangeaux : essai sur la chronologie de Grégoire de Tours, en Mélanges de l'École française de Rome, 94/2 (1982).

- La ville de Tours du IV au VI siècle: naissance d'une cité chrétienne, Roma, 1983.

- Quand et comment Martin de Tours est-il devenu le saint patron par excellence de la Gaule ?, en Jean-Pierre CAILlet et al. (eds.), Des dieux civiques aux saints patrons (IVe-VII ${ }^{e}$ siècle), París, 2015.

PIETRI, Luce y HeIjMAns, Marc, Gregorius Turonensis, en Prosopographie chrétienne du BasEmpire, t. 4, vol. 1, París, 2013.

Rousselle, Aline, Croire et guérir. La foi en Gaule dans l'Antiquité tardive, París, 1990.

SCHLICK, J., Composition et chronologie des De virtutibus sancti Martini de Grégoire de Tours, en Studia Patristica, VII (1966).

SHAW, Richard, Cbronology, Composition, and Authorial Conception in the Miracula, en Alexander Callander MURRAY (ed.), A Companion to Gregory of Tours, Leiden-Boston, 2015.

UYTFANGHE, Marc van, La controverse biblique et patristique autour du miracle, et ses répercussions sur l'hagiographie dans l'Antiquité tardive et le haut Moyen Âge latin, en Hagiographie, cultures et sociétés. IV $V^{e}$-XII siècles. Actes du Colloque organisé à Nanterre et à Paris (2-5 mai 1979), París, 1981.

VOGÜÉ, Adalbert de, Histoire littéraire du mouvement monastique dans l'Antiquité, tome. IV. Première partie: le monachisme latin, París, 1997.

WOOD, Ian, The Individuality of Gregory of Tours, en Kathleen MITCHELL y Ian WOOD (eds.), The World of Gregory of Tours, Leiden-Boston, 2002. 